\title{
Evaluation of a Novel Calibration Technique for Optically Tracked Oblique Laparoscopes
}

\author{
Stijn De Buck ${ }^{1}$, Frederik Maes ${ }^{1}$, André D'Hoore ${ }^{2}$, and Paul Suetens ${ }^{1}$ \\ ${ }^{1}$ Faculties of Medicine and Engineering, Medical Image Computing (ESAT and \\ Radiology), K.U.Leuven, Herestraat 49, 3000 Leuven, Belgium \\ 2 Department of Abdominal Surgery, UZ Leuven, Herestraat 49, 3000 Leuven, \\ Belgium \\ stijn.debuck@uz.kuleuven.ac.be*
}

\begin{abstract}
This paper proposes an evaluation of a novel calibration method for an optically tracked oblique laparoscope. We present the necessary tools to track an oblique scope and a camera model which includes changes to the intrinsic camera parameters thereby extending previously proposed methods. Because oblique scopes offer a wide 'virtual' view on the surgical field, the method is of great interest for augmented reality guidance of laparoscopic interventions using an oblique scope.

The model and an approximated version are evaluated in an extensive validation study. Using 5 sets of 40 calibration images, we compare both camera models (i.e. model and approximation) and 2 interpolation schemes. The selected model and interpolation scheme reaches an average accuracy of 2.60 pixel and an equivalent 3D error of $0.60 \mathrm{~mm}$.

Finally, we present initial experience of the presented approach with an oblique scope and optical tracking in a clinical setup. During a laparoscopic rectum resection surgery the setup was used to augment the scene with a model of the pelvis. The method worked properly and the attached probes did not interfere with normal procedure.
\end{abstract}

\section{Introduction}

Laparoscopic techniques evolve very rapidly through a broad spectrum of enhancements [1] like the introduction of stereoscopic lenses, so called 'chip in the tip' lenses [1], etc. .. One of these enhancements is the use of scopes which have a viewing direction different from the mechanical rotation axis of the lens. Using such a scope holds the advantage of having a broader virtual field of view due to the easy rotation and the inclination. This property is considered a valuable asset in, for instance, laparoscopic rectum surgery and should thus be taken into account. In 2] Yamaguchi et al. introduced a camera model for an oblique (or tilted view angle) scope of which the angle is determined by a rotary encoder. The model tries to incorporate the physical behaviour of such a camera and introduces possible deviations from the ideal model. The resulting calibration

\footnotetext{
* We acknowledge the support of the IWT/OZM050811 project.
} 
method is accurate but very specific to oblique scopes. It also does not incorporate changes to the camera intrinsic parameters.

In this paper we will present an accuracy evaluation of a novel calibration technique enabling the use of a tilted view angle laparoscope tracked only by an optical tracker. We extend the work of Yamaguchi et al. 2] by incorporating changes to the intrinsic camera parameters which renders the method more generic. Furthermore, we make use only of optical tracking for measuring the pose of the camera and lens thereby reducing hardware requirements as compared to the setup in 2. This approach enlarges the application domain since it facilitates tracking of multiple bodies such as other instruments besides the laparoscopic camera (e.g. tracked ultrasound devices, ...). In fact, it provides a common reference frame in which multiple applications, with different requirements can be realized. The presented calibration approach can be applied when an oblique scope is used in such a generic setup. However, the use of an optical tracker will have an impact on the accuracy results. Therefore we conducted an extensive accuracy study of the proposed calibration approach.

\section{Materials and Methods}

The setup we use, consists of a CCD-camera, a $30^{\circ}$ view angle laparoscope, an optical tracker (Flashpoint 5000, Image guided technologies) and an Octane workstation (SGI). Both the lens and the CCD-camera are equipped with IRLEDs visible to the optical tracker (see Fig. 1) and can both be located accurately within the coordinate space of the optical tracker. In order to augment a laparoscope video with preoperative images we should know the parameters of the imaging system for each position and orientation of both lens and camera. Before we describe the computation of the parameters modeling the rotation of the oblique lens, we will present the camera model and its relation to the optical tracking device and explain how the angle $\theta$ between the CCD-camera and the lens can be determined.

\subsection{Camera Model}

A conventional straight laparoscope can be modeled sufficiently accurately by a pinhole model in conjunction with a radial distortion component [3]. The perspective model and radial distortion can be described by

$$
\left[\begin{array}{c}
\lambda p_{i}^{(u)} \\
\lambda
\end{array}\right]=K R\left(P_{i}-C\right) \text { and } \quad\left\|p_{i}^{(u)}-c\right\|=(1+\kappa)\left\|p_{i}^{(d)}-c\right\|
$$

In these equations $P_{i}$ is a point in world coordinates, $p_{i}^{(d)}, p_{i}^{(u)}$ are its corresponding image points, after and before distortion respectively. $c\left(C_{x}, C_{y}\right)$ is the center of distortion. The rotation matrix $R$ and translation vector $C$ relate the world coordinate system to the camera coordinate system. The projection matrix $K$ projects the transformed point onto the undistorted image which is distorted using a radial distortion parameter $\kappa$. 
Supposing that the tracker coordinate system coincides with the world coordinate system and we know $C$ and $R$ for one position ref of the scope, the projection in any other position $l$ can be computed as:

$$
\left[\begin{array}{c}
\lambda p_{i}^{(u)} \\
\lambda
\end{array}\right]=K\left[\begin{array}{ll}
I_{3} & 0
\end{array}\right] T_{\text {cam }} T_{\text {Tracker }-L P}^{(l)}\left[\begin{array}{c}
P_{i} \\
1
\end{array}\right]
$$

in which $T_{\text {Tracker }-L P}$ is the matrix relating the tracker coordinate frame $O_{\text {Tracker }}$ to a local coordinate system of the camera IR-LEDs $O_{L P}, I_{3}$ is a unit matrix of size 3 and $T_{\text {cam }}$ can be computed as follows

$$
T_{\text {cam }}=\left[\begin{array}{cc}
R-R C \\
0 & 1
\end{array}\right] T_{\text {Tracker }-L P}^{(\text {ref }}{ }^{-1}
$$

and remains constant as long as the IR-LEDs are rigidly attached to the camera. This model applies to any orientation of an oblique scope as long as the camera head is not rotated about the lens axis.

Each orientation differs by the fact of having a different image plane. In an ideal oblique scope this difference is due to a rotation about the mechanical axis of the lens and, thus, the camera center $C$ remains fixed. Because of lens imperfections both the center and the intrinsic parameters contained in $K$ might change whenever the lens is rotated with respect to the camera head. Whereas others (e.g. 4) use affine transformation to model variations in $R$ and $K$, we propose a projective transformation or homography $H$. Changes in $C$ can be modeled by a translation correction term. The projection of point $P_{i}$ for an arbitrary orientation $l$ of the lens w.r.t. the CCD-head about an angle $\theta$ can be written as follows:

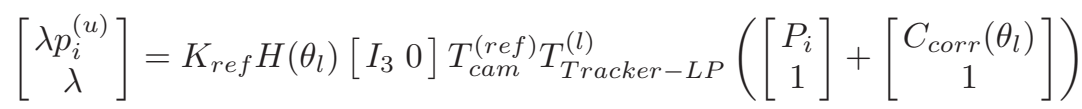

in which $K_{\text {ref }}$ contains the parameters modeling a chosen reference orientation and $T_{\text {cam }}^{(r e f)}$ is computed based on the same reference set. Since we have a pure rotation of the lens, one could omit $C_{\text {corr }}(\theta)$.

The intrinsic and extrinsic parameters of Eq. 1 for a straight lens or scope in a fixed position can be computed by means of a calibration jig (Fig. 1), that has a known geometry in the tracker reference frame. The centers of the discs are detected fully automatically and assigned to their proper 3D coordinates. After selection of a reference image, one can compute $K_{r e f}, R_{r e f}, C_{r e f}$ and thus $T_{\text {cam }}^{(r e f)}$ based on the 2D-3D pairs 53 .

\subsection{Computation of Theta}

The angle $\theta$ between the CCD-camera and the oblique lens can be computed on the basis of the relative pose of the IR-LEDs attached to the lens and the CCD-camera. In a first stage the invariant axis for a rotation of the camera head about the lens is computed. The second stage then determines the angle between measured poses about the invariant rotation axis. 

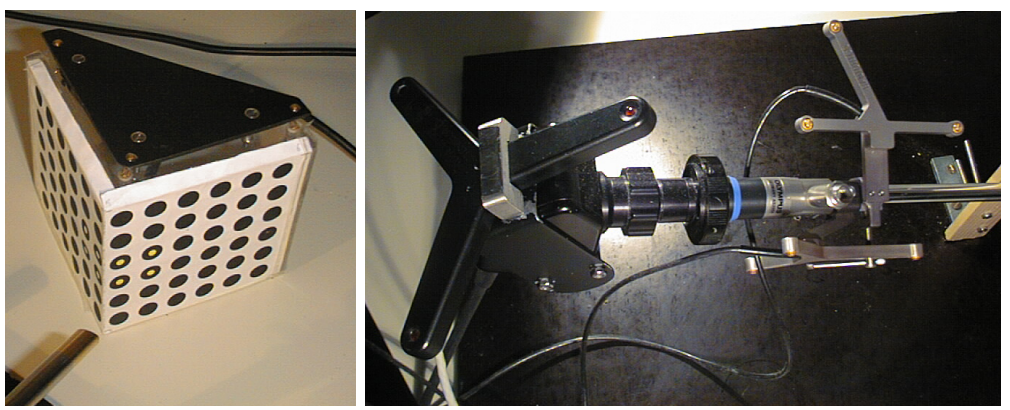

Fig. 1. Picture of the setup used for the calibration of an oblique scope. This includes the in house developed calibration jig with 3 attached IR-LEDs. An additional IR-LED probe was attached to the oblique lens, which is used to compute the angle between the lens and the CCD-camera.

\subsection{Computation of the Center Correction Term and the Homography}

Before computing the homography $H(\theta)$, we can compute the correction term $C_{\text {corr }}(\theta)$ by imposing that the camera center of a calibration image $l$ should equal the reference camera center after applying the correction term. After constructing a set of $C_{c o r r}^{(l)}$ for each recorded pose of the oblique scope at $\theta_{l}$, we can build a function $C_{\text {corr }}(\theta)$ by interpolation.

For the computation of $H_{l}$ we acquire a set of images covering the range of orientations of camera and lens that is expected during the procedure. In order to keep the effect of measurement noise from the optical tracker minimal, the lens and jig remain fixed at one position while the CCD-head is rotated. The disc centers for all images are detected automatically and corresponding centers of the reference image are assigned. Subsequently, we can compute the homography $H_{l}$ of each image $l$ by finding the homography that maps

$$
\left[\begin{array}{ll}
I_{3} & 0
\end{array}\right] T_{\text {cam }}^{(r e f)} T_{\text {Tracker-LP }}^{(l)}\left(\left[\begin{array}{c}
P_{i} \\
1
\end{array}\right]+C_{\text {corr }}(\theta)\right) \quad \text { onto } \quad K_{\text {ref }}^{-1} p_{i}^{(u)}
$$

For the computation we use a method by Horaud et al. [6].

Next, one can compute the parameters of a function $H(\theta)$ interpolating $H_{i}$ which enables the computation of the homography for an arbitrary $\theta$.

\subsection{Interpolation of the Parameters}

To be able to use the camera model of an oblique scope for arbitrary angles, we need to construct a function for both the homography $H(\theta)$ and the center correction $C \operatorname{corr}(\theta)$. Two interpolation schemes were developed and tested: a truncated Fourier series

$$
f(\theta)=\frac{a_{0}}{2}+\Sigma_{k=1}^{2} a_{k} \sin (k \theta)+b_{k} \cos (k \theta)
$$

and a polynomial of an empirically determined fifth degree. 


\section{Experiments}

\subsection{Accuracy Assessment}

In order to assess the accuracy of the approach and compare the two interpolation schemes, we performed a validation experiment on 4 sets of 40 images. The experiment was conducted as follows:

- First 5 times 40 images were acquired of the calibration jig with the lens in the same position and the camera head each time slightly rotated about the lens. Position and orientation of both lens and camera head were recorded by the optical tracker.

- Next, a calibration was computed for all (Sets A-E) of the 5 acquired sets while one set was used to validate (set A). Note that the reported results for set $\mathrm{A}$ depict a residue instead of a true validation.

- The image plane error $\epsilon_{l}$ was computed for each image $l$ as

$$
\epsilon_{l}=\frac{1}{n} \sum_{i=1}^{n}\left\|\widehat{p_{i, l}}-p_{i, l}\right\|
$$

with

$$
\left[\begin{array}{c}
\lambda \widehat{p_{i, l}} \\
\lambda
\end{array}\right]=K_{\text {ref }} H\left(\theta_{l}\right)\left[\begin{array}{ll}
I_{3} & 0
\end{array}\right] T_{\text {cam }}^{(r e f)} T_{\text {Tracker-LP }}^{(l)}\left(\left[\begin{array}{c}
P_{i} \\
1
\end{array}\right]+\left[\begin{array}{c}
C_{\text {corr }}\left(\theta_{l}\right) \\
1
\end{array}\right]\right)
$$

and $p_{i, l}$ equal to the image coordinate of the detected corresponding disc center and $P_{i}$ the $3 \mathrm{D}$ coordinate of the disc center. $n$ equals the number of detected disc centers for each image. Furthermore, a 3D equivalent error was computed as the distance between the back-projected ray through a disc center $p_{i, l}$ and the corresponding $3 \mathrm{D}$ point $P_{i}$.

This experiment was repeated for each calibration set altering both the interpolation scheme from a Fourier series to a polynomial interpolation and the camera model from the complete model including the center correction $C_{\text {corr }}(\theta)$ to the one neglecting this term.

A typical result from one set (set $\mathrm{C}$ ) is plotted in Fig. 2 which visualizes the error as a function of $\theta$. The resulting error function seems independent of $\theta$.

The result of all experiments is summarized in Tab. 1 and 2 , For each set (B-E) we show the mean and standard deviation of the error over all images of that set. The results are given for the 4 experiment types numbered as below:

1 no center correction \& Fourier interpolation (NCC-FI)

2 no center correction \& polynomial interpolation (NCC-PI).

3 center correction \& Fourier interpolation (CC-FI).

4 center correction \& polynomial interpolation (CC-PI).

Tab. 1 shows the image plane error in pixel while Tab. 2 shows the 3D error in $\mathrm{mm}$. The latter is an objective way to represent the error as it is independent of the camera geometry. It does depend on the distance between the jig and the camera which we chose in the range expected in a real surgical setting. 

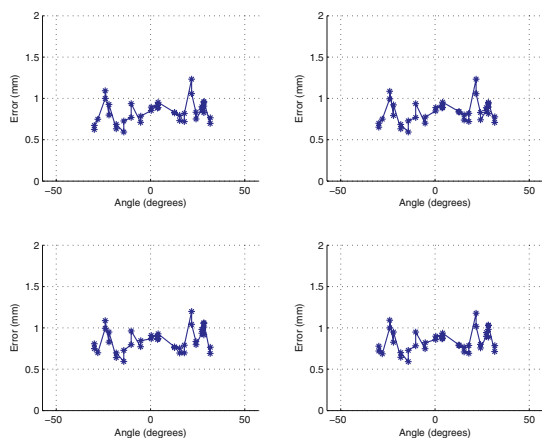

Fig. 2. A plot of the computed error in pixels where estimated projections of points are compared to detected ones. The 4 plots denote the result as a function of $\theta$ for the four types of modeling/interpolation. (top left = NCC-FI; top right $=$ NCC-PI; bottom left $=$ CC-FI; bottom right $=$ CC-PI)

Table 1. Summary of the validation results for 5 sets. Each row represents the 2D image plane error in pixels computed on set A after calibration and interpolation using sets A-E and each considering a different combination of interpolating function and camera model (See Sec. 3). The average error over all experiments (sets B-E) is given in the last column.

\begin{tabular}{|c||c|c|c|c||c|}
\hline & $\mathrm{B}$ & $\mathrm{C}$ & $\mathrm{D}$ & $\mathrm{E}$ & Average \\
\hline NCC-FI & $2.62 \pm 0.96$ & $3.58 \pm 0.56$ & $1.77 \pm 0.79$ & $2.41 \pm 1.03$ & $2.60 \pm 0.84$ \\
NCC-PI & $2.62 \pm 0.96$ & $3.58 \pm 0.54$ & $1.79 \pm 0.82$ & $2.44 \pm 1.06$ & $2.61 \pm 0.85$ \\
CC-FI & $2.54 \pm 1.03$ & $3.64 \pm 0.57$ & $1.78 \pm 0.72$ & $2.45 \pm 0.89$ & $2.60 \pm 0.80$ \\
CC-PI & $2.55 \pm 1.03$ & $3.60 \pm 0.56$ & $1.78 \pm 0.74$ & $2.62 \pm 1.10$ & $2.64 \pm 0.86$ \\
\hline
\end{tabular}

From the results in Tab. 1 and 2, we can not deduce a significant difference between the polynomial and Fourier series interpolation. Because of its inherent periodicity and equally good performance, we chose to use the truncated Fourier interpolation.

As for the inclusion of $C_{c o r r}$, the experiment shows an increased error when adding this term to the model, although this difference is not significant. The increased error is probably due to the noise of the optical tracker measurement which leads to errors on the computed rotation angles $\theta$. The use of a more complex model encompasses more degrees of freedom which, in the absence of a clear translation component, may merely model noise that is incorporated in the interpolation function. When evaluating a control set, the model will also render noisy estimates resulting in a higher error. This is confirmed by comparing the residue for set A shown in Tab. 3 before and after interpolation. Before interpolation the inclusion of $C_{c o r r}$ results in a slightly higher accuracy. However, after interpolation, there is no significant difference noticeable.

Since the neglect of the term results in a model with fewer parameters which is less prone to noise and in a higher accuracy, we propose to use the approximation. 
Table 2. Summary of the calibration results for the 5 sets (A-E) which were validated on set A. Each row represents the 3D error in mm computed on set A after calibration and interpolation and each considering a different combination of interpolating function and camera model (See Section 3). The average error over all experiments (sets B-E) is given in the last column.

\begin{tabular}{|c||c|c|c|c||c|}
\hline & $\mathrm{B}$ & $\mathrm{C}$ & $\mathrm{D}$ & $\mathrm{E}$ & Average \\
\hline NCC-FI & $0.62 \pm 0.22$ & $0.83 \pm 0.13$ & $0.41 \pm 0.18$ & $0.56 \pm 0.23$ & $0.60 \pm 0.19$ \\
NCC-PI & $0.61 \pm 0.22$ & $0.83 \pm 0.13$ & $0.41 \pm 0.18$ & $0.57 \pm 0.24$ & $0.61 \pm 0.19$ \\
CC-FI & $0.59 \pm 0.24$ & $0.85 \pm 0.14$ & $0.41 \pm 0.17$ & $0.57 \pm 0.21$ & $0.61 \pm 0.19$ \\
CC-PI & $0.60 \pm 0.24$ & $0.84 \pm 0.13$ & $0.41 \pm 0.17$ & $0.61 \pm 0.26$ & $0.61 \pm 0.20$ \\
\hline
\end{tabular}

Table 3. Residue of obtained by calibration of set A. Both the residue before and after interpolation are provided. The left column shows the $2 \mathrm{D}$ errors in pixel while the right column shows the $3 \mathrm{D}$ error in millimeter. This table shows that incorporating a center correction results in a more accurate model and that after interpolation the improvement is canceled out. This is probably due to measurement noise on the IRLEDs.

\begin{tabular}{|l||c|c|}
\hline & 2D residue (pixel) & 3D residue (mm) \\
\hline No interpolation/no center corr & $0.81 \pm 0.11$ & $0.19 \pm 0.03$ \\
No interpolation/center corr & $0.72 \pm 0.03$ & $0.17 \pm 0.01$ \\
NCC-FI & $1.48 \pm 0.72$ & $0.35 \pm 0.17$ \\
NCC-PI & $1.46 \pm 0.64$ & $0.34 \pm 0.15$ \\
CC-FI & $1.49 \pm 0.73$ & $0.35 \pm 0.18$ \\
CC-PI & $1.42 \pm 0.59$ & $0.33 \pm 0.15$ \\
\hline
\end{tabular}

\subsection{Clinical Usability}

We also tested if the proposed method was usable in a clinical context. During a routine laparoscopic intervention in which an oblique scope is employed, we displayed a model of the pelvis on the acquired laparoscopic video stream. Calibration was performed as described above.

During the intervention, the sterilized lens was reattached to the CCD-head which was wrapped in sterile transparent plastic. The display of the virtual model on top of the real video stream worked well: under the used range of orientations, the model remained visibly stationary with respect to the patient. During the usage, the operator had to pay attention that the IR-LEDs were visible to the optical tracker, though once he found a comfortable position, this was easily asserted. Except for the wrapping of the CCD camera, which took slightly more time due to the size of the IR-LED probe, no significant delays in the surgical workflow were noted.

\subsection{Discussion}

By combining conventional calibration techniques, tracking of both lens and camera and a mapping technique based on homographies, we model the behaviour 
of a rotating oblique lens and can estimate the projection parameters for an arbitrary position of camera and lens. By providing a model for a lens based system that can cope with both changes to extrinsic and intrinsic parameters, we extend the work of Yamaguchi et al. 2]. Whereas they use a robotic device in their approach, we consider only optical tracking for obtaining pose data. That approach can be an advantage in the design of an (augmented reality) image-guided surgery system since it facilitates the tracking and representation of other devices (e.g. instruments) in a single reference frame.

Validation of this model and comparison with a simplified version on an extensive set of images, shows that for the application of oblique scopes we can neglect the center correction term $C_{\text {corr }}$ without losing accuracy. This makes the model easier to update and less prone to noise. In our first clinical tests the calibration seems to work well and does not interrupt normal work flow.

The accuracy may be improved by reducing the influence of the IR-LED measurement noise on the computation of $\theta$. This may be realized by the development of a dedicated circular IR-LED probe. This probe could consist of a circular array of IR-LEDs mounted around the mechanical rotation axis of the oblique lens such that it would present itself in a well conditioned way to the optical tracker, independent of the orientation of the probe.

\section{References}

1. Boppart, S.A., Deutsch, T.F., Rattner, D.W.: Optical imaging technology in minimally invasive surgery. Current status and future directions. Surgical Endoscopy 13(7), 718-722 (1999)

2. Yamaguchi, T., Nakamoto, M., Sato, Y., Nakajima, Y., Konishi, K., Hashizume, M., Nishii, T., Sugano, N., Yoshikawa, H., Yonenobu, K., Tamura1, S.: Camera model and calibration procedure for oblique-viewing endoscope. In: Ellis, R.E., Peters, T.M. (eds.) MICCAI 2003. LNCS, vol. 2879, pp. 373-381. Springer, Heidelberg (2003)

3. De Buck, S., Van Cleynenbreugel, J., Geys, I., Koninckx, T., Koninckx, P.R., Suetens, P.: A system to support laparoscopic surgery by augmented reality visualization. In: Niessen, W.J., Viergever, M.A. (eds.) MICCAI 2001. LNCS, vol. 2208, pp. 691-698. Springer, Heidelberg (2001)

4. Simon, G., Berger, M.O.: Registration with a zoom lens camera for augmented reality applications. In: IWAR 1999, p. 103. IEEE Computer Society, Washington, DC, USA (1999)

5. Tsai, R.: An efficient and accurate camera calibration technique for 3D machine vision. In: CVPR 1986, pp. 364-374 (1986)

6. Horaud, R., Csurka, G.: Self-calibration and euclidean reconstruction using motions of a stereo rig. In: ICCV 1998 pp. 96-103 (1998) 